\title{
The Auger@TA Project: Phase II Progress and Plans
} \author{
Auger $^{5, *}$ and Telescope Array ${ }^{8, * *}$ collaborations \\ ${ }^{1}$ Dept of Physics, Case Western Reserve University, Cleveland OH (USA) \\ ${ }^{2}$ Institute for Cosmic-Ray Research, University of Tokyo, Tokyo (Japan) \\ ${ }^{3}$ Dept of Physics, Colorado School of Mines, Golden CO (USA) \\ ${ }^{4}$ Dept of Physics \& Astronomy, University of California - Los Angeles, Los Angeles CA (USA) \\ ${ }^{5}$ Pierre Auger Observatory, Malargüe (Argentina) \\ Full author list: http://www.auger.org/archive/authors_2018_10.html \\ ${ }^{6}$ Karlsruhe Institute of Technology, Karlsruhe (Germany) \\ ${ }^{7}$ Dept of Physics, Sungkyunkwan University, Jang-an-gu, Suwon (Korea) \\ ${ }^{8}$ Telescope Array Project, Salt Lake City UT (USA) \\ Full author list: http://www.telescopearray.org/research/collaborators
}

Corbin Covault ${ }^{1}$, Toshihiro Fujii ${ }^{2}$, Robert Halliday ${ }^{1}$, Jeffrey Johnsen ${ }^{3}$, Ryan Lorek $^{1}$, Toshiyuki Nonaka ${ }^{2}$, Sean Quinn $^{1,4}$, Hiroyuki Sagawa ${ }^{2}$, Takashi $\mathrm{Sako}^{2}$, Fred Sarazin ${ }^{3}$, Ricardo Sato ${ }^{5}$, David Schmidt ${ }^{6}$, and Ryuji Takeishi ${ }^{7}$ for the Pierre

\begin{abstract}
The Auger@TA project is a combined effort involving members of both the Pierre Auger Observatory and the Telescope Array experiment (TA) to cross-calibrate detectors and compare results on air showers detected at one location. We have recently reported results from Phase I of the project, during which we collected and presented data from two Auger water Cherenkov surface detector stations deployed into the TA experiment near the Central Laser Facility. For Phase II, we will deploy a micro-array of six or seven single-PMT Auger surface detector stations co-located with TA scintillator surface detector stations. The Auger micro-array will trigger and collect data independently from the TA allowing for a complete end-to-end comparison of detector data, calibration, and reconstructed event quantities on a shower-by-shower basis between the TA and Auger detector systems. We describe progress towards development of the micro-array for Phase II including the preparation of surface detector water tanks, station electronics, wireless communications, triggers, and data acquisition. We also outline plans for deploying the Auger@TA micro-array into the TA experiment in 2019 with preliminary estimates for coincident air-shower rates.
\end{abstract}

\section{Introduction}

The Pierre Auger Observatory (Auger) [1] and the Telescope Array (TA) [? ] are the two major observatories for the highest energy cosmic rays. Auger is located near Malargüe, Argentina and views the southern sky. TA is located near Delta, Utah, USA, and views the northern sky. Both Auger and TA are hybrid observatories, combining atmospheric fluorescence telescopes with large arrays of surface detectors sensitive to shower particles arriving at the ground. However, Auger and TA use different surface detector materials; Auger surface detector (SD) stations are water Cherenkov detectors (WCD), while TA SD stations use scintillator panels. Both media are widely used in particle physics but have different sensitivity to $\mu, e$, and $\gamma$ channels at varying distance from the shower core.

During the past several years, the Auger and TA collaborations have each reported results for measurements on energy spectra, anisotropy and composition for cosmic rays in the energy range above about $10^{18} \mathrm{eV}$. While the

\footnotetext{
*e-mail: auger_spokespersons@fnal.gov

**e-mail: jui@cosmic.utah.edu
}

Auger and TA results are largely in agreement, there exists some tension between the two in certain areas. For example, while the Auger and TA all-particle cosmic ray spectra agree up to $\approx 50 \mathrm{EeV}$, the results begin to diverge at the highest energies [3]. This discrepancy could be due to experimental difference in Auger vs. TA that might impact the measurement of cosmic rays. Or it could be due different astrophysics scenarios that might come into play if there are differences in the source distributions for the northern vs. the southern hemispheres.

The Auger@TA project is designed to investigate instrumental energy-dependent effects via an in situ crosscalibration between SD stations, accomplished using a two-phase approach: Phase I activities are largely complete, with preliminary results summarized in section 2 and in detail elsewhere in this symposium [4]. Phase II activities will begin in early 2019. Table 1 lists the major activities associated with each of the two phases. The primary aspect of Phase I was the deployment of two Auger WCD surface detector stations at the TA Central Laser Facility (CLF). The primary aspect of Phase II will be the 
Table 1. Phase I and Phase II activities for Auger@TA

\begin{tabular}{ll}
\hline \hline Phase I & $\begin{array}{l}\text { Deployed Auger South \& North WCD } \\
\text { surface detector stations into TA at CLF. }\end{array}$ \\
\cline { 2 - 2 } & $\begin{array}{l}\text { Verified hardware level triggers between } \\
\text { TA and Auger doublet. }\end{array}$ \\
& Verified integrated signals of Auger doublet. \\
\cline { 2 - 2 } & $\begin{array}{l}\text { Translated between TA (MIP) } \\
\text { and Auger (VEM) signals. }\end{array}$ \\
\hline \hline Phase II & $\begin{array}{l}\text { Deployment of 6 or 7 Auger WCD into an } \\
\text { independent 'micro-array' within TA. }\end{array}$ \\
& $\begin{array}{l}\text { Dual reconstruction with Auger } \\
\text { WCDs and TA SD signals. }\end{array}$ \\
& $\begin{array}{l}\text { Separate reconstruction using WCD } \\
\text { and TA SD signals (allows for } \\
\text { cross-check of reconstruction methods). }\end{array}$ \\
\hline \hline
\end{tabular}

deployment of a micro-array of six or seven Auger WCDs into TA.

\section{Overview of Phase I:}

Figure 1 shows a recent (June 2018) panoramic photo of the equipment located at the TA Central Laser Facility (CLF) corresponding to Auger@TA Phase I. We use four detectors at the the CLF: one Auger South style station (identical to those deployed in Argentina), one Auger North prototype station, and two TA SD stations. The northwest TA SD station (local trigger) serves as a local trigger system for the Auger doublet. The southwest TA SD station (DET2427) participates in the TA global trigger, allowing a direct comparison between Auger and TA traces for events near the CLF.

Figure 2 shows a closer photo of the two Auger WCD tanks deployed at the TA CLF corresponding to Phase I for Auger@TA. The far tank is the Auger South style surface detector (SD) with three PMTs. The near tank is an Auger North style prototype station which is very similar to the Auger South station but with a single PMT and somewhat enhanced electronics.

During Phase I we have initiated "doublet" studies comparing the signal responses between the Auger South WCD and the Auger North WCD. For Phase II we will use Auger North WCDs which were previously deployed in Colorado. As indicated in Figure 3, there is good correlation between the signal response of both types of WCD stations. Grey dots represent local coincidences (not fully reconstructed) while black dots represent showers that are also triggered and reconstructed by TA. This demonstrates that we can use Auger North surface detector tanks as a well-calibrated proxy for Auger South detectors that comprise the Auger Observatory in Argentina. Additional studies comparing the response of the Auger SD to TA SD are described in detail elsewhere [4].

\section{Pierre Auger and Telescope Array surface detector stations}

The ultimate goal of the Auger@TA project is event-byevent cross-calibration between Auger and TA detectors. Figure 4 shows the basic structure of both the Auger and TA surface detector stations.

The Auger SD station is a Water Cherenkov Detector that collects light from its water volume. Phototube traces are digitized and analyzed by station-level trigger algorithms. Signals are calibrated in terms of $I_{V E M}$, the intensity from one Vertical Equivalent Muon (VEM). Events with a signal $>3.2 I_{V E M}$ correspond to a level-2 trigger (T2) and are saved locally. T2 timestamps are reported by each station at roughly $23 \mathrm{~Hz}$. A central data acquisition system sends a readout request if adjacent stations see correlated $\mathrm{T} 2$ events in certain geometries. Calibration is performed online and the previous minute's calibration data is provided with every readout request [1?].

The TA SD station detects secondary particles via fluorescence light emitted the plastic scintillator. Phototube waveforms are digitized and analyzed by local station electronics. Any signal $>0.3$ minimum ionizing particles (MIP) (Level 0 trigger) is stored in memory at the station while any signal $>3$ MIPS is promoted (Level 1 trigger) and sent to the central system to form an array trigger the station Level 1 trigger rate is about $30 \mathrm{~Hz}$. Calibration data is provided for 10 minute periods. The linearity of PMTs is calibrated using an LED flasher arrangement [5].

\section{Phase II: An Auger Micro-array within TA}

Figure 5 shows a plan view of the existing TA (upper left) and zoomed inset (lower right) corresponding to one planned deployment option for six Auger North style WCD stations to locations immediately adjacent to existing TA stations at the positions indicated (yellow pins). We have already examined satellite surveys for these locations which all provide reasonable access to nearby gravel roads for water delivery.

More recently we are considering the option of deploying Auger stations into a triangular array corresponding to the exact geometry and spacing of the Auger Observatory in Argentina. Such a configuration would allow for a more seamless application of Auger reconstruction techniques. In this case, up to eight Auger stations may be deployed. possibly re-deploying stations currently located at the CLF. A final decision for station positions for the Phase II micro-array is pending further study.

Figure 6 is a photo of six Auger North type WCD stations stored at the TA Cosmic Ray Center in Delta, Utah. The six stations are instrumented and ready for deployment into the TA.

\subsection{Micro-Array Telecommunications}

Wireless telecommunications are needed for the sixstation Auger micro-array, as shown schematically in Figure 7. We will use commercial off-the-shelf (COTS) 


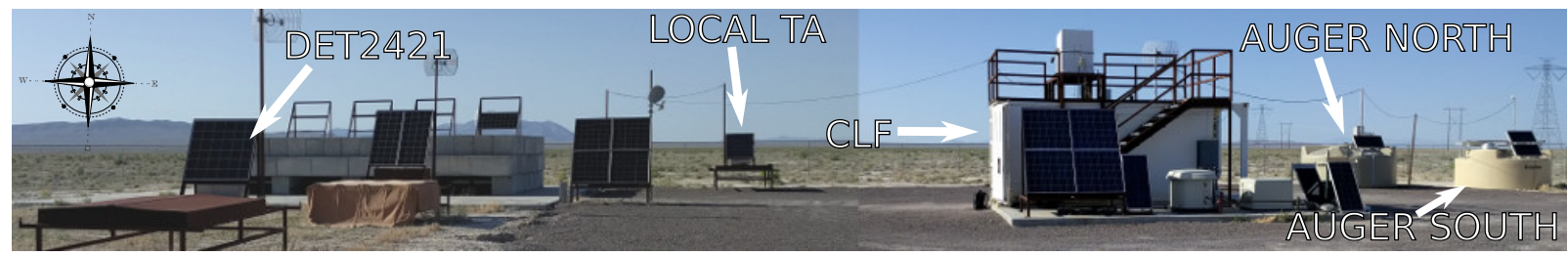

Figure 1. Phase I of Auger@TA at the TA Central Laser Facility (CLF) indicating TA stations DET2421 (part of the global TA array), a local TA station, and two Auger water Cherenkov detector (WCD) surface detector (SD) stations (Auger North and Auger South).

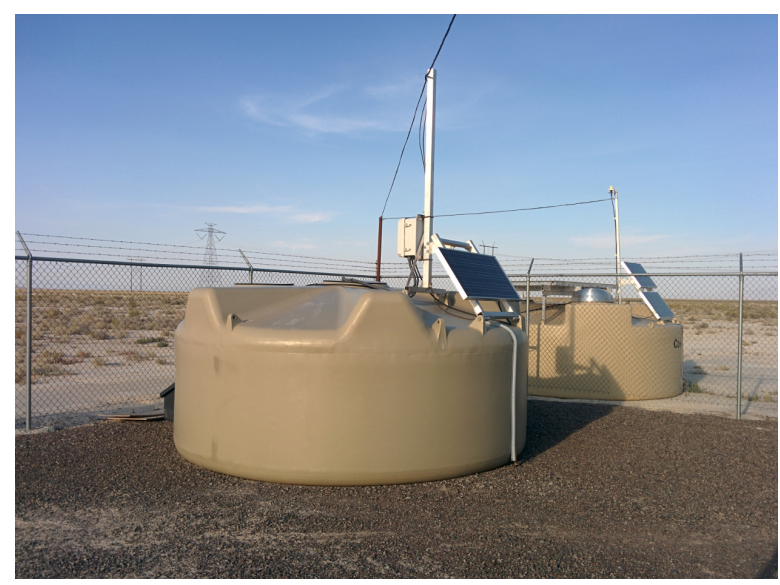

Figure 2. Two Auger Water Cherenkov Detector (WCD) surface detector stations deployed at the Central Laser Facility (CLF) of the Telescope Array (TA) corresponding to Phase I for Auger@TA: Auger North (near) and Auger South (far).

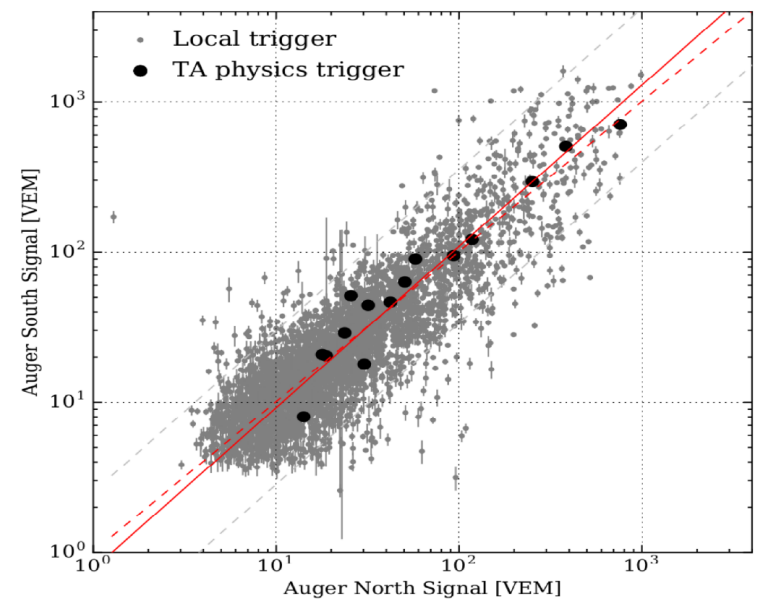

Figure 3. WCD response: Auger South vs. Auger North WCD. Grey dots indicate local triggers (unreconstructed showers, solid red line power-law slope $=1.1 \pm 0.1)$ while black dots correspond to showers that also trigger the Telescope Array (reconstructed showers, dashed red line power-law slope $=1.01 \pm 0.02$ ). This good correlation will allow us to use Auger North stations within the TA as proxies for Auger South stations already deployed at the Pierre Auger Observatory in Argentina.
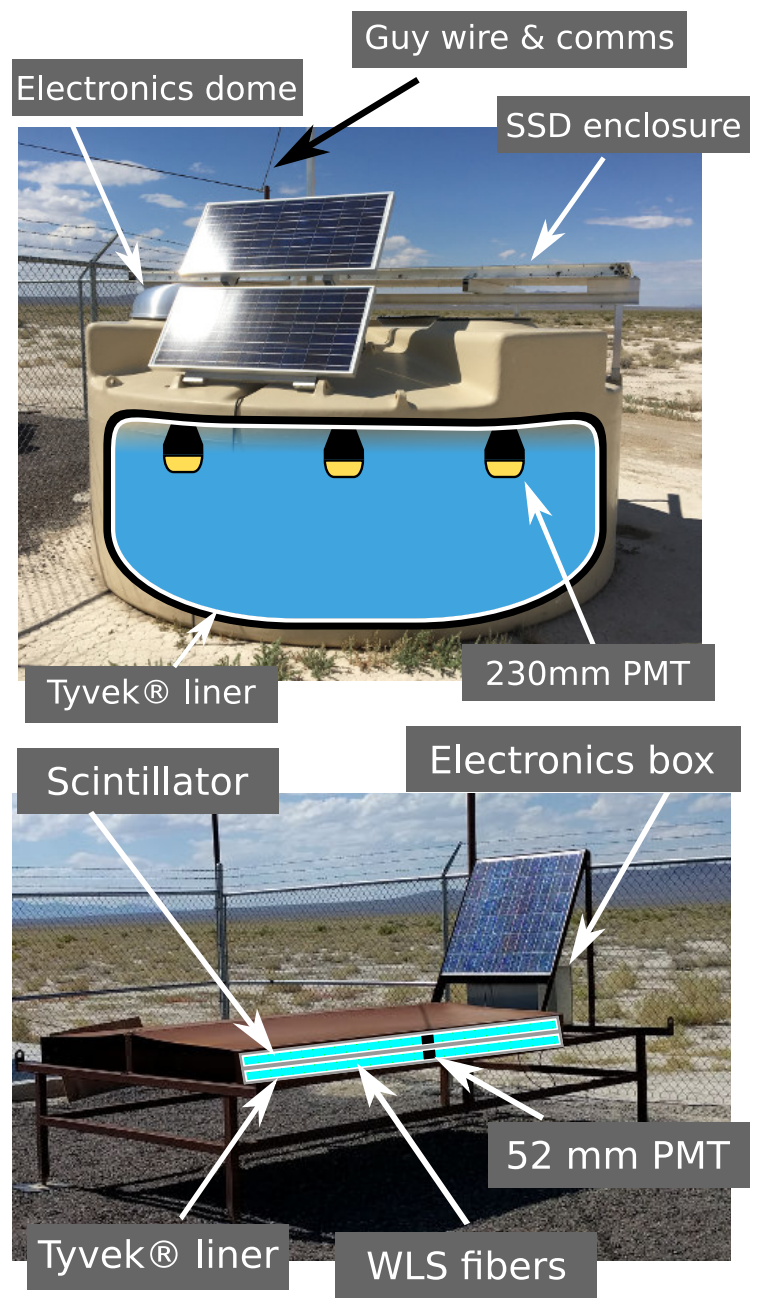

Figure 4. Auger Water Cherenkov Detector station (top) vs. Telescope Array Scintillator Detector station (bottom).

point-to-point communications based on the Digi Xbee $900 \mathrm{MHz}$ RF modem and associated $13 \mathrm{~dB}$ HG913Y Yagi antennas. Xbee modems will be interfaced to each Auger SD local station controller and to a micro-array Central Data Acquisition System (CDAS) located at the TA radio tower. Each communication modem link will be controlled and monitored at each end using customprogrammed Raspberry Pi processors.

We have already field-tested a two-point bi-directional version of the communications system with data transfer rates of up to $10 \mathrm{kpbs}$ over a distance of $10.9 \mathrm{~km}$, (1024- 


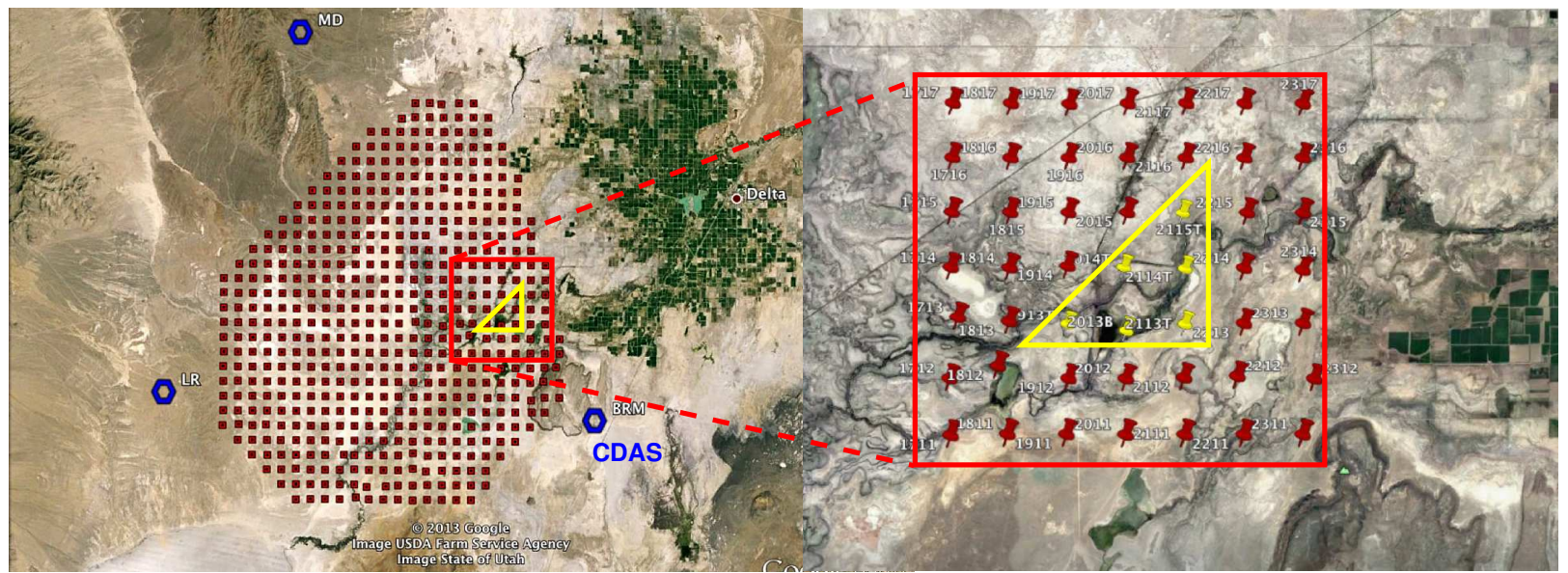

Figure 5. Plan view of Telescope Array surface detector stations (red pins) with inset indicating the positions where the Auger@TA Phase II micro-array of six Auger WCDs (yellow pins) will be co-located. Auger station positions correspond to one possible configuration under consideration. Also indicated (blue hexagons) are the locations of TA radio towers, one of which is co-located with the planned Central Data Acquisition Systems (CDAS) for the micro-array. (The image above was made using Google Earth and includes components from http://www.physics.utah.edu/ bergman/telescope_array.html, used with permission.)

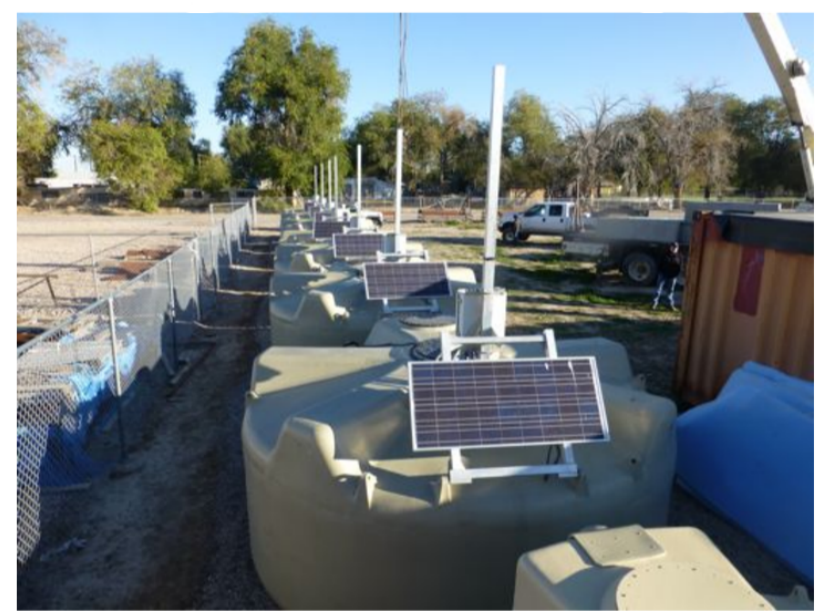

Figure 6. Auger North style WCD tanks ready for deployment into the Auger@TA Phase II micro-array.

byte packets) with zero measured unrecoverable data loss over $\sim 2.5$ hour transmission test within the TA.

\subsection{Predicted Event Rates}

For Phase II, the advent of the Auger micro-array will allow for independent and/or combined triggering and event reconstruction for both Auger and TA subsystems. Events within the TA are $50 \%$ efficient at about $10^{18.2} \mathrm{eV}$ and fully efficient at just below about $10^{19} \mathrm{eV}$ [? ]. Figure 8 shows a flowchart of the anticipated analysis chain for Phase II. Estimated rates for showers that can be independently reconstructed by both TA (high quality events with at least 5 stations) and the Auger micro-array (at least 3 stations) range from about 5 events per month (with core fully-contained and reconstructed within Auger micro-array) to about 1015 events per month (core contained within and provided by TA only).

\section{Conclusions}

The Auger@TA project has successfully installed and completed initial analysis of data from the first phase of a collaborative experimental effort to provide an in situ cross-calibration between Auger and Telescope Array surface detector stations. A joint Working Group of collaborators from both Auger and TA is now moving to complete Phase II of the project. We will deploy a micro-array of six Auger surface detectors into the operating Telescope Array which will allow air shower events to be simultaneously and independently detected and reconstructed by by both detector systems. Such an approach should allow for accurate and detailed cross-checks between Auger and TA from the level of raw detector response up through the analysis chain including details of event reconstruction. Current plans call for the deployment of the Phase II micro-array by early 2019 . 


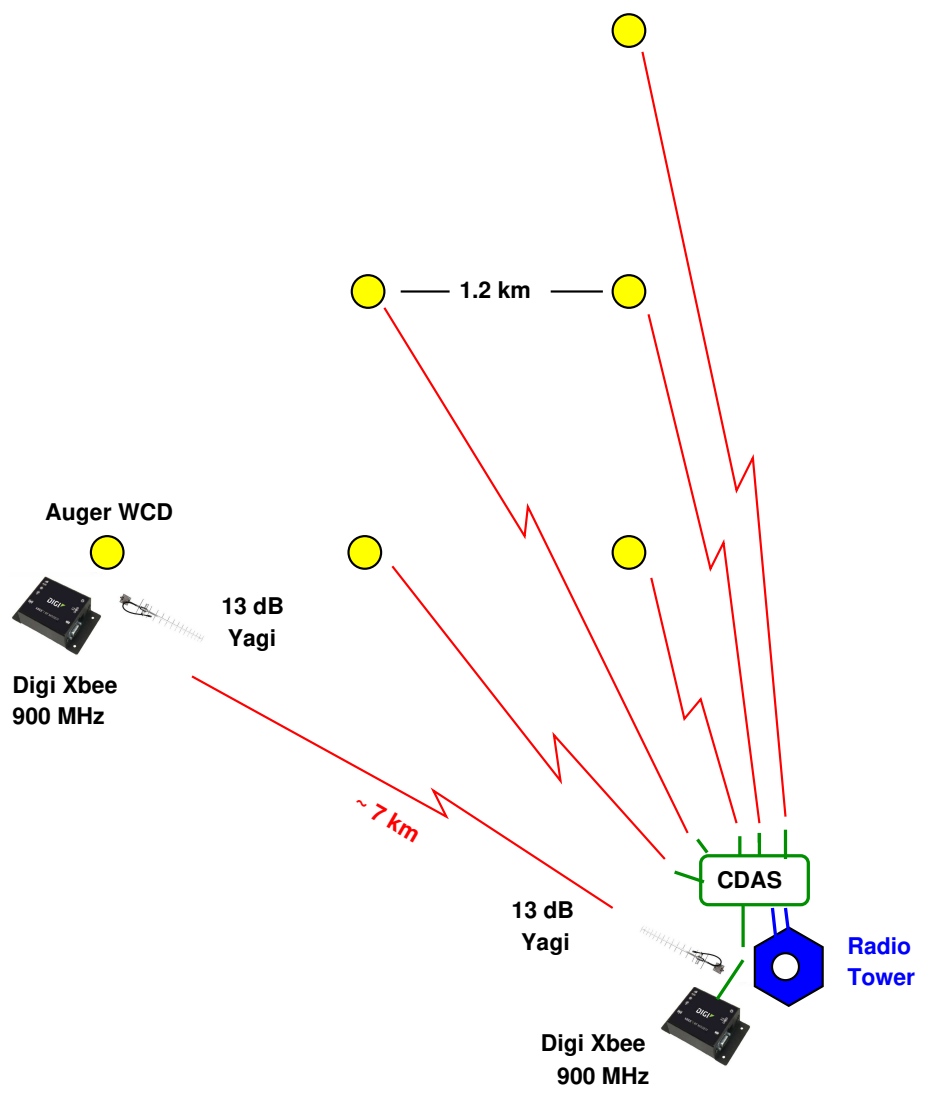

Figure 7. Schematic view of planned telecommunication strategy for the Phase II Auger@TA micro-array.

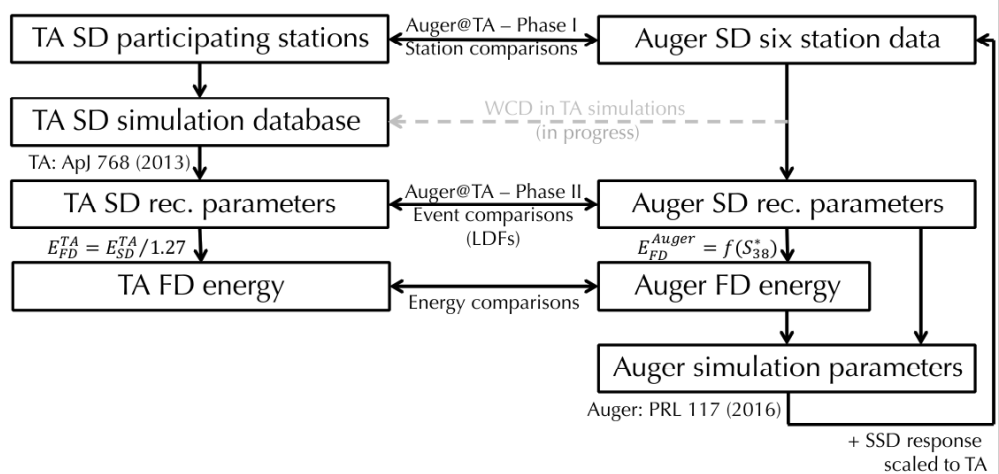

Figure 8. Phase II analysis chain.

\section{Acknowledgments}

This project was supported in part by JSPS Grant-in-Aid for Scientific Research (A) 25247035 and the National Science Foundation. CEC is grateful for support from CWRU. We thank the Pierre Auger \& Telescope Array collaboration for their continued support.

\section{References}

[1] A. Aab et al. [Pierre Auger Collaboration], "The Pierre Auger Cosmic Ray Observatory", NIM A798
172 (2015).

[2] T. Abu-Zayyad et al. [Telescope Array Collaboration], "The surface detector array of the Telescope Array experiment", NIM A 689, 87 (2012).

[3] C. Patrignani et al. (Particle Data Group), Chin. Phys. C 40, 100001 (2016)

[4] F. Sarazin, (joint Auger@TA working group), these proceedings.

[5] Telescope Array Collab. ApJ 768, L1, (2013) 